\title{
Seeded growth of monodisperse gibbsite platelets to adjustable sizes
}

\author{
Judith E.G.J. Wijnhoven \\ Van't Hoff Laboratory for Physical and Colloid Chemistry, Debye Institute, Utrecht University, Padualaan 8, NL-3584 CH Utrecht, The Netherlands
}

Received 19 August 2004; accepted 3 June 2005

Available online 18 July 2005

\begin{abstract}
Nanosized hexagonal gibbsite seeds are grown from a mixture of dissolved alumina alkoxides at $85^{\circ} \mathrm{C}$. Centrifugation reduces the polydispersity by $30 \%$. The seeds can be grown further by adding them to a fresh alkoxide mixture and heating it. This procedure was repeated several times to obtain particles of $570 \mathrm{~nm} \pm 11 \%$ diameter and a thickness of $47 \pm 23 \%$. No indications of a size limit were observed. The thus obtained particles may form easily a columnar phase. Individual gibbsite particles in solution can be seen by confocal microscope.

(C) 2005 Elsevier Inc. All rights reserved.
\end{abstract}

Keywords: Colloidal platelets; Monodisperse; Gibbsite; Seeded growth; Confocal microscopy

\section{Introduction}

Gibbsite, $\gamma-\mathrm{Al}(\mathrm{OH})_{3}$, has been studied for at least three reasons. Firstly, it is an important semi-manufacture product from the Bayer process in the aluminum production [1-3]. Studies on Bayer liquors are largely motivated by the importance to improve the crystallization. Gibbsite particles obtained from Bayer liquors have particles size ranging from a few micrometers to $150 \mu \mathrm{m}$. The shape differs; lozenges, hexagons, prisms, and their aggregates are the most frequently observed [4]. Secondly, gibbsite is studied for applications as a product as such: as polishing agent in toothpaste [5,6], as a fire retardant [5], as coating and filler in paper manufacturing [6], or as rheology enhancer in drilling muds [7]. And thirdly, since gibbsite is an important part of soils, people have studied its surface chemistry extensively [8-11].

Nanosized gibbsite particles are synthesized according to a few different methods [9,12-17]. These methods have in common that gibbsite is formed at moderate $\mathrm{pH}$ (4.5-9), in contrast to the Bayer process, where the crystallization takes place at very high $\mathrm{pH}$.

Here, a mixture of aluminum alkoxides dissolved in diluted $\mathrm{HCl}$ is used [17]. It produces fairly monodisperse gibb-

E-mail address: j.e.g.j.wijnhoven@chem.uu.nl. site platelets with an average diameter of $150-200 \mathrm{~nm}$. The size of the particles can be varied slightly by varying the reaction conditions. Particles prepared following this method are used as model colloids for studies on dynamics $[18,19]$ and phase behavior of platelets [20-22].

In order to further study the phase behavior and dynamics of these particles, it is necessary to be able to manipulate their size and to reduce their polydispersity. Simple methods to achieve these goals, i.e., centrifugation to lower polydispersity and seeded growth to increase size are reported. The potential of these new particles for further fundamental colloidal studies is also demonstrated.

\section{Experimental}

All chemicals are used as received from the supplier. Gibbsite seeds are synthesized according to the literature [16,17] with slight modifications: $0.08 \mathrm{~mol}, 19.7 \mathrm{~g}$ aluminum secondary butoxide (ASB, $\geqslant 95 \%$, Fluka) and $0.08 \mathrm{~mol}, 16.3 \mathrm{~g}$ aluminum isopropoxide (AIP, 98+\%, Acros) are dissolved in $1 \mathrm{~L} 0.09 \mathrm{M}$ hydrochloric acid ( $\mathrm{HCl}$ $37 \%$, p.a., Merck) solution by stirring for $7-10$ days. The resulting mixture, which varies from clear to turbid, is heated in a polyethylene bottle in a water bath at $85^{\circ} \mathrm{C}$ for $72 \mathrm{~h}$. After cooling to room temperature, the dispersion is dialyzed 
1 week against a continuous flow of demineralized water in regenerated cellulose tubes (Visking, MWCO 12,00014,000).

To reduce the polydispersity and to concentrate the particles, dispersions are centrifuged at $1100 \mathrm{~g}$ in glass bottles until the supernatant is bluish (overnight, 15-20 h). The sediment is easily redispersed by stirring, either in water to store the particles or in an ASB/AIP mixture to use them as seeds.

One half to two third (4-6 g/L for the first step) of the product of a synthesis is used as seeds for the following growing step (which is at the same scale). Sedimented seeds are redispersed in a fresh solution of dissolved alkoxides ASB/AIP. The mixture is heated at $85^{\circ} \mathrm{C}$ for $72 \mathrm{~h}$ in an oil bath and is stirred to prevent the particles from sedimentation. It is important that through the whole sample seeds are available, otherwise a secondary nucleation of gibbsite takes place. For particles with a diameter larger than $350 \mathrm{~nm}$ stirring should be very vigorous. Mechanical stirring is needed, since the use of a magnetic stirring bar is not sufficient: particles sediment. After growth, the resulting particles are treated as above: dialysis against demineralized water for 1 week and centrifugation to remove small particles. During dialysis, the suspensions are mixed once or twice a day by turning the dialysis tubes to avoid sedimentation and consequently slow dialysis. The centrifugation is done at lower $g$ forces than for the seeds because large particles sediment faster. In general, centrifugation should take $15-20 \mathrm{~h}$. Too fast centrifugation may cause aggregation. The sequence of sedimentation-growth-dialysis is repeated four times.

Particle diameters are determined from images made by use of a Tecnai 12 (FEI Company) transmission electron microscope (TEM) operated at $120 \mathrm{kV}$. The area of at least 100 particles is measured with the help of an imageprocessing program (AnalySIS Pro, Soft Imaging Systems). From the area, the diameter is calculated assuming the particles are perfect hexagons. Atomic force microscopy (AFM) is used to determine the thickness of the particles. A suspension of gibbsite is spread over a freshly cleaved mica substrate and dried in air. AFM measurements (more than 100 per sample, except for the largest particles where only 11 measurements were possible due to the tendency of the particles to group on the substate) are done in tapping mode on a MultiMode scanning probe microscope (Digital Instruments) with a standard TESP silicon tip (Digital Instruments). The largest particles are imaged using a Nikon Eclipse TE2000 confocal scanning laser microscope (CSLM) in reflection mode.

By X-ray diffraction the crystallinity of the particles was tested. Room temperature XRD measurements on dry powder are carried out on a Bruker-Nonius CPS 120 powder diffractometer system, equipped with a Inel position sensitive detector with $2 \theta$ up to $120^{\circ}$. The radiation used is $\operatorname{Co} K \alpha_{1}, \lambda=1.78897 \AA$. High resolution XRD on aqueous suspensions are also performed at Swiss-Norwegian beam line BM- 1 at the ESRF, Grenoble, France, with $\lambda=0.375 \AA$.

\section{Results and discussion}

\subsection{Reducing polydispersity by centrifugation}

During synthesis of colloids, unwanted material like aggregates or secondary nucleation particles might be formed. Centrifugation has proven to be a suitable technique to remove aggregates [23]. Centrifugation is also applied as a method to remove unwanted small material like particles from a secondary nucleation in seeded growth procedures [24]. In that case the supernatant contains the waste. Here, centrifugation has proven to be a quick method to reduce the high polydispersity of a sample. Small and light particles remain in the supernatant whereas large particles (the sample) sediment.

A gibbsite dispersion obtained directly after synthesis and dialysis is used as starting material (Fig. 1a). Such a system is polydisperse and contains unidentified material in between

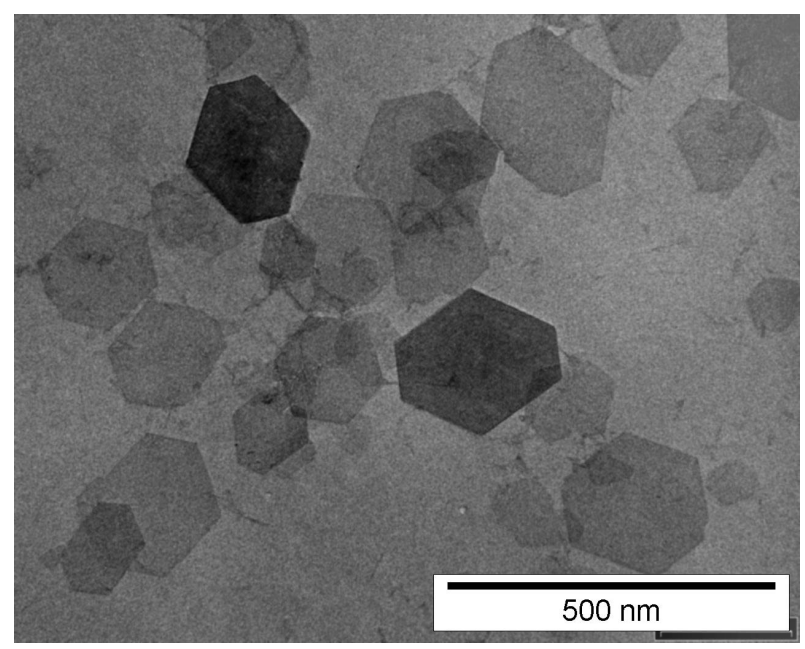

(a)

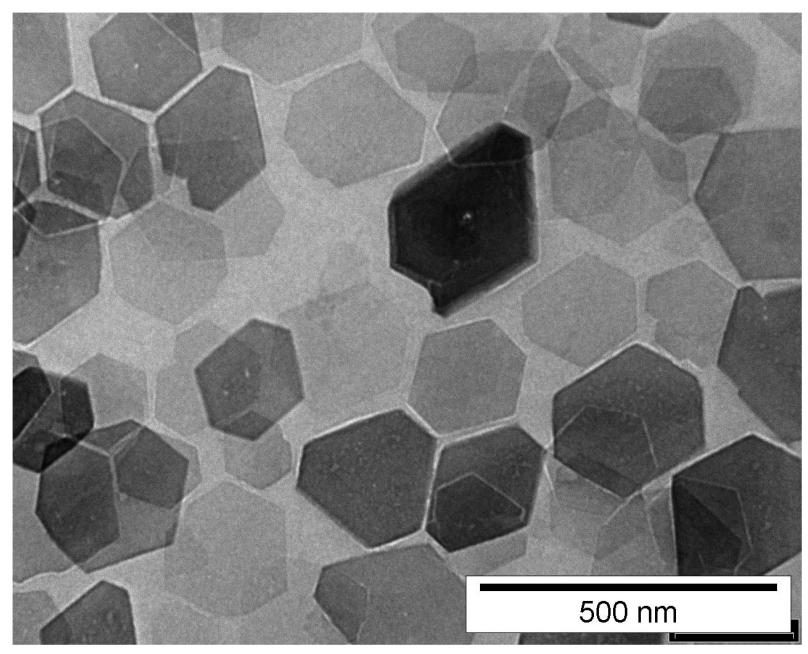

(b)

Fig. 1. TEM images. (a) Hexagonal gibbsite platelets directly after synthesis and dialysis, diameter is $170 \mathrm{~nm} \pm 30 \%$. Small platelets and unidentified material are also visible. (b) Gibbsite platelets after short centrifugation of the batch of Fig. 1a, diameter is $220 \mathrm{~nm} \pm 21 \%$. 
the gibbsite hexagons. The average size of the particles is $170 \mathrm{~nm} \pm 30 \%$, as determined by TEM. After centrifugation for $7.5 \mathrm{~h}$ at $330 \mathrm{~g}$ a fraction with a size of $220 \mathrm{~nm} \pm 21 \%$ has been sedimented (Fig. 1b). Not only the polydispersity is reduced but the average particle size is also increased by centrifugation. Since the supernatant is still turbid, it is centrifuged further at a higher speed and for a longer period: $910 \mathrm{~g}$ and $48 \mathrm{~h}$. The sediment contains gibbsite particles of $170 \mathrm{~nm} \pm 20 \%$. Even after this period the supernatant is not clear but still slightly turbid.

\subsection{Tailor made sizes}

The increase of the size range of gibbsite particles has been subject of a few small tests in our laboratory [25]. Reaction conditions have been varied: the composition of the reaction mixture, stirring time before heating, reaction temperature, and reaction time. Although small influences are observed, no significant larger gibbsite plates were obtained. Particles can also be formed at room temperature (Fig. 2). The reaction is very slow (about a year) and particles do not grow to large sizes. All in all, reaction at room temperature turns out to be a method to obtain smaller gibbsite particles; on average a diameter of $90 \mathrm{~nm} \pm 30 \%$. The particles are so small because a very large number of seeds is formed.

As it is known from literature [16], tetraethoxy silane (TEOS) influences the formation of boehmite. The needles are elongated by the addition of TEOS, presumably because the TEOS attaches selectively to certain lattice planes of the crystal promoting the growth of the uncovered faces. Here the addition of TEOS on the formation of gibbsite is investigated. Experiments were performed in five steps with concentrations of TEOS of $0.8,1.6,2.4,3.2$, and $4.0 \mathrm{~g} / \mathrm{L}$. As can be seen from Fig. 3, the addition of TEOS causes the formation of long structures, likely boehmite rods, amid the gibbsite particles. The gibbsite formation itself is also

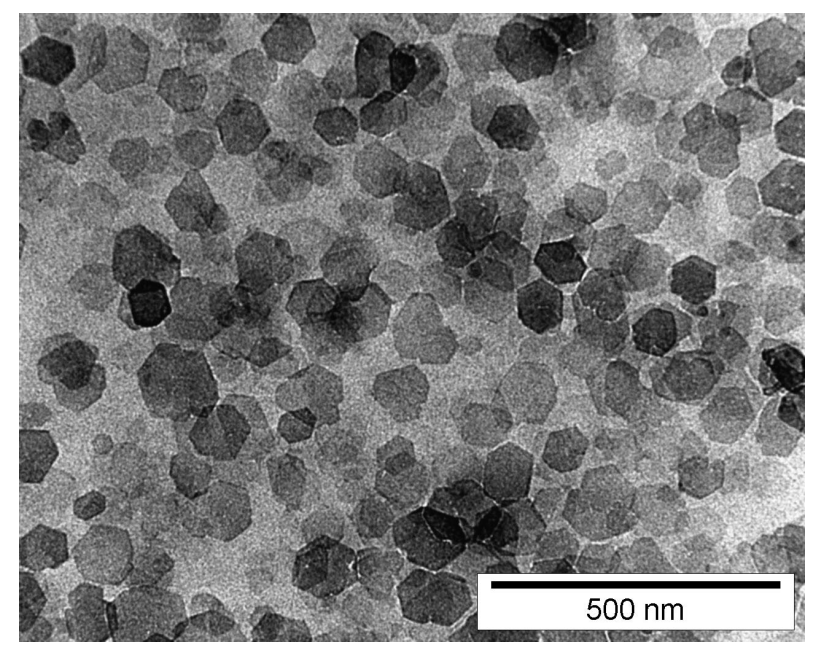

Fig. 2. TEM images of small gibbsite platelets formed at room temperature in three years, diameter is $90 \mathrm{~nm} \pm 30 \%$. influenced. By increasing TEOS concentration, the size of the gibbsite particles increases whilst the polydispersity decreases, both by $10 \%$. Because of the coprecipitation of other materials and the slight influence on the particle size, the method has not been studied further.

From the foregoing it can be concluded that the most promising method to obtain larger crystals is the seeded growth. Seeded growth is applied frequently for colloids. Spherical particles like amorphous silica [26,27] or polycrystalline iron [28] are used in seeded growth procedures, but also crystalline particles like gibbsite in the Bayer process [2] are suitable for seeded growth. Gibbsite particles are used as seeds in a fresh mixture of alkoxides. In Fig. 4a the resulting particles from a seeded growth of gibbsite seeds of $210 \mathrm{~nm} \pm 19 \%$ in four growth steps to particles of $570 \mathrm{~nm} \pm 11 \%$ are shown. The single particles spread nicely over the grid; they are not aggregated. They show, however, tendency to lie on top of each other. Their hexagonal shape maintains during growth as expected and particles do not grow together in aggregates as is it seen in the Bayer process.

Closer inspection of the particles learns that the edges of the largest particles do not look the same as for the smaller particles (see Fig. 5b). The shape of the sides seems not flat but pointed. In the last growing step less seeds than before were used leading to a large increase in size and a higher growth rate. Probably the higher growth rate causes the less sharp edges. The effect is only observed in this step.

In-house $\mathrm{X}$-ray diffraction (XRD) measurements in a wide range of diffraction angles $2 \theta$ confirmed the material is gibbsite. For a more detailed analysis of the peak width with synchrotron radiation, a smaller $2 \theta$ range containing (002), (110), and (200) XRD reflections was selected (Fig. 5a). The (002) reflection originates from the atomic structure along the platelet normal and is clearly broadened well beyond the instrument resolution due to the finite particle thickness. The experimentally determined pro-

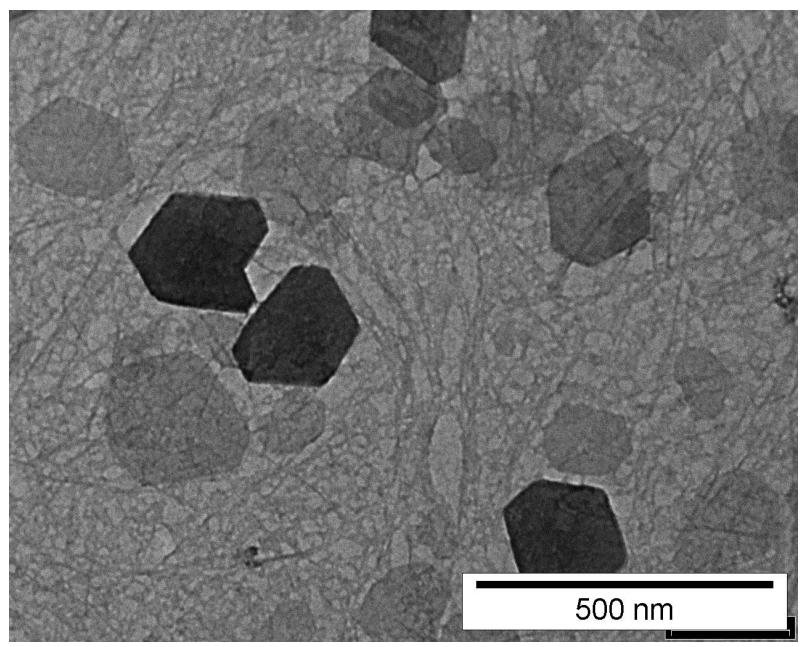

Fig. 3. TEM images of gibbsite platelets formed in the presence of tetraethoxy silane $(2.4 \mathrm{~g} / \mathrm{L})$. Presumably boehmite needles coprecipitate. 


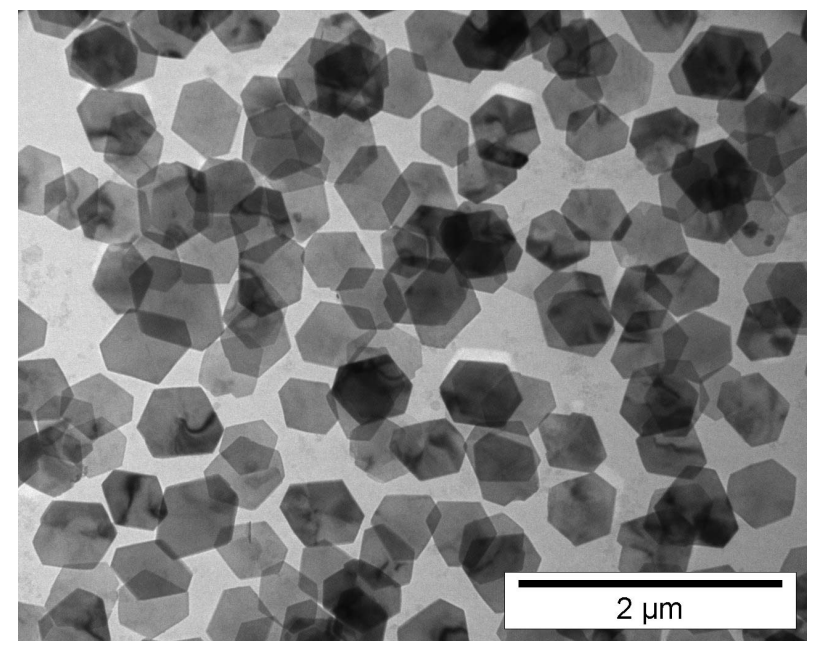

(a)

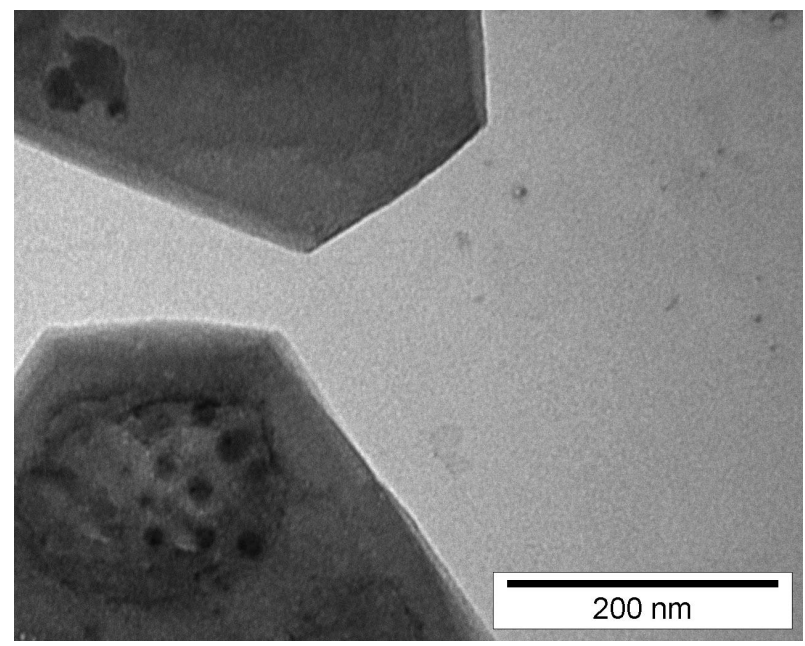

(b)

Fig. 4. (a) TEM of monodisperse gibbsite particles from a seeded growth procedure (4 growing steps), diameter is $570 \mathrm{~nm} \pm 11 \%$. (b) Enlargement of sides of the particles of Fig. 4a to show the different shape of the sides.

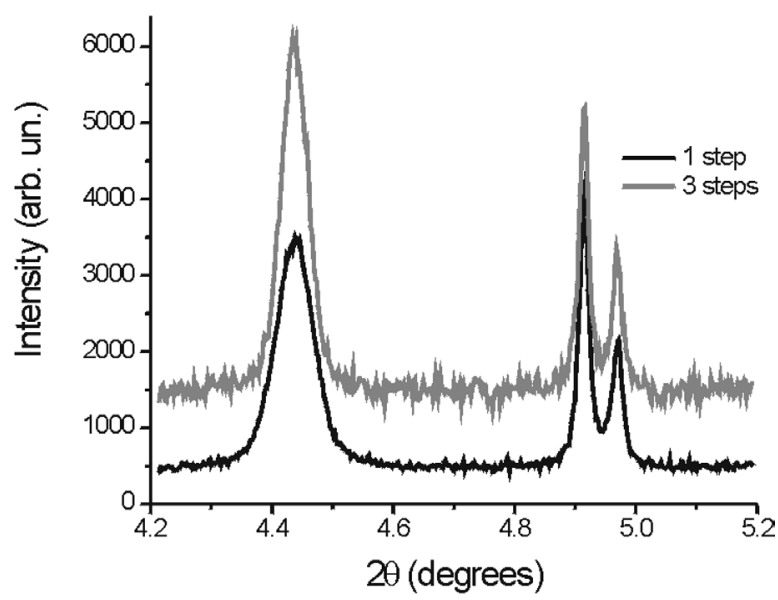

(a)

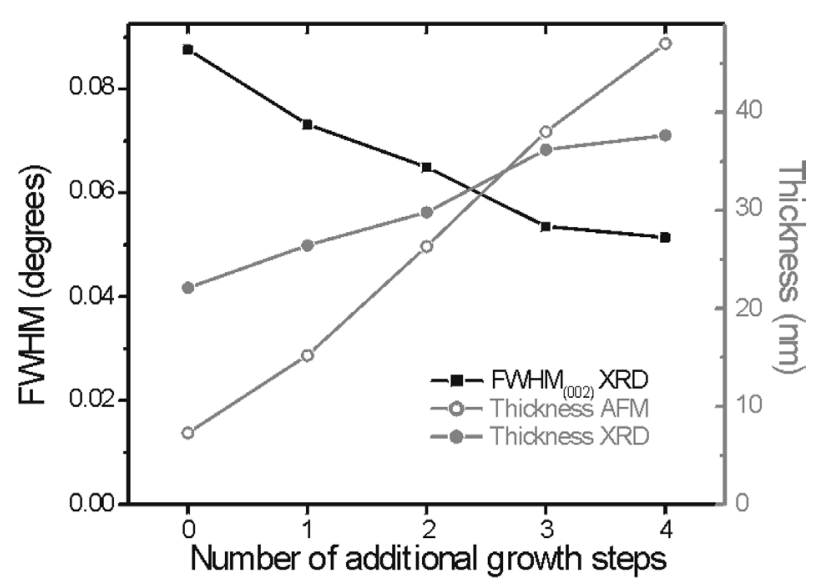

(b)

Fig. 5. (a) Examples of high-resolution XRD spectra obtained from a suspension of the particles after 1 and 3 additional growth steps. The background (mostly originating from the solvent) was fit to a second-order polynomial and subtracted from the data. The spectra are normalized and displaced vertically for clarity (by 500 and 1500 units, respectively). (b) Development of the FWHM of the (002) reflection with growth steps (0 corresponds to the seed particles). The AFM and XRD-derived particle thickness are also given (gray lines).

file of the (002) reflection was fitted to a Gaussian. The full-width-at-half-maximum $\left(\mathrm{FWHM}_{(002)}\right)$ of the fits is displayed in Fig. 5b. The latter was used to estimate the particle thickness $t$ using the Debye-Scherrer formula [29], $t=0.9 \lambda /\left(\mathrm{FWHM}_{(002)} \cos \theta\right)$. One can see that in comparison with the AFM results the XRD data significantly overestimate the thickness of the seed particles and the particles after one additional growth step. This discrepancy can be (partially) explained by a very large thickness polydispersity of the particles. The contribution of a particle to XRD is proportional to its volume, which might enhance the role of the largest particles and, therefore, larger value of $t$. This effect can be further enhanced by correlation between the particle thickness and the diameter.

Additional growth steps are seen to lead to sharper (002) $\mathrm{XRD}$ powder reflection. This suggests that the atomic layers of the seed and the additionally grown layers are correlated. Yet, the peak width reduces by less than a factor of 2 while the AFM data indicate a seven-fold increase of the thickness. This can be partially caused by a reduction of the thickness polydispersity of the platelets, which can reduce the discussed above overestimation of $t$ by the Debye-Scherrer formula. Moreover, the difference in the AFM and XRD estimation of the thickness can also indicate that the atomic layers of the seed and the additional layers are not always correlated.

In Fig. 5a, the (110) and (200) reflections arise from the atomic structure along the platelets. The present data do not allow for quantitative analysis of the intrinsic width of the (110) and (200) reflections, which are mainly instrumentlimited. Yet, we have observed a reduction of their apparent width with increasing size. This suggests a certain degree of 
correlation of the atomic lattices in the directions parallel to the platelets as well.

An overview of the particle diameter and thickness of all the intermediate steps is given in Table 1. In the first three steps, the aspect ratio of the particles decreases during growth. This means that the different crystal planes do not grow at the same rate. This was also observed for circumstances comparable to the Bayer process: Sweegers et al. [4] studied the strong influence of the growth conditions on the particle shape and aspect ratio. It is expected that the aspect ratio in our system can also be influenced by working at other growth conditions. An indication for this expectation is that in the last step, where the growth conditions were different (lower seed concentration), the aspect ratio did not change. The different growth conditions apparently lead to a more regular growth in both the thickness and the diameter of the particle.

The higher the number of seeds, the lower the increase in size and vice versa. To try to find the limit of low seed concentration and thus the largest size increase per step, a three times lower seed concentration was tested. Particles were grown from 220 to $360 \mathrm{~nm}$ in one step with a little secondary-nucleation particles (Fig. 6). The secondary nucleation is due to the lack of stirring during growth. Without secondary nucleation the size increase of the seeds would have been even larger. Particles grown at such a high growth rate are not so well shaped. They start to deviate from the

Table 1

Dimensions of the synthesized particles

\begin{tabular}{llcc}
\hline Step & Diameter (TEM) & Thickness (AFM) & $L / D$ \\
\hline 0 & $210 \mathrm{~nm} \pm 19 \%$ & $7 \mathrm{~nm} \pm 52 \%$ & 0.03 \\
1 & $270 \mathrm{~nm} \pm 18 \%$ & $15 \mathrm{~nm} \pm 48 \%$ & 0.06 \\
2 & $350 \mathrm{~nm} \pm 14 \%$ & $26 \mathrm{~nm} \pm 43 \%$ & 0.07 \\
3 & $420 \mathrm{~nm} \pm 15 \%$ & $38 \mathrm{~nm} \pm 36 \%$ & 0.09 \\
4 & $570 \mathrm{~nm} \pm 11 \%$ & $47 \mathrm{~nm} \pm 23 \%$ & 0.08 \\
\hline
\end{tabular}

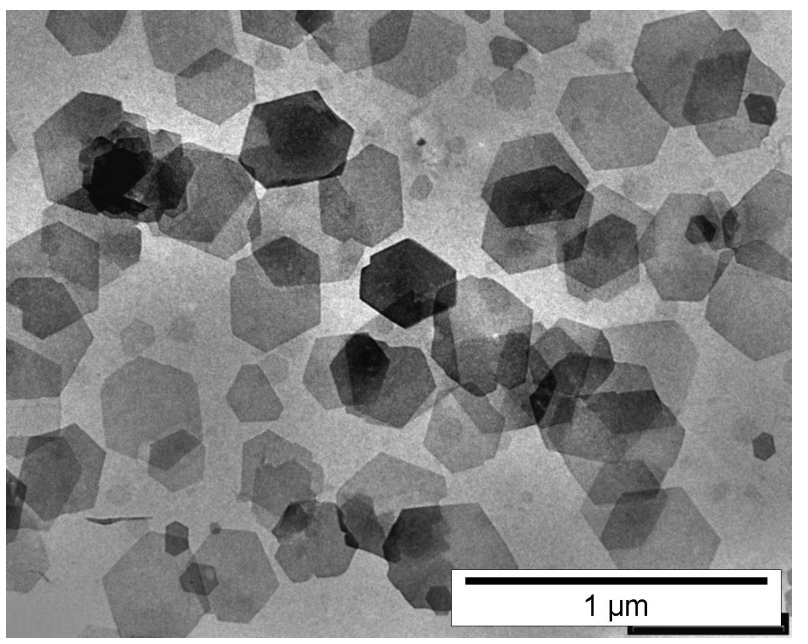

Fig. 6. TEM images of a bidisperse sample obtained from a seeded growth procedure with low seed concentration without stirring, diameter of the large particles is $360 \mathrm{~nm}$. hexagonal form as can be seen in Fig. 6. The characteristic abnormality in the platelets are notches in the side planes. The notches are also found in small amounts in the other growth steps. Due to the other growth conditions other planes of the crystal might be favored to grow [4].

\subsection{Potential use}

The possibilities to use monodisperse tailor made gibbsite platelets are numerous. A study of platelet phase behavior depending on size and ionic strength will be presented in another paper [29]. Two preliminary experiments with the prepared monodisperse large platelets are presented here: formation of a columnar phase and visibility in a confocal microscope.

In the columnar phase, platelets order in stacks [22]. In Fig. 7a, a schematic image of the columnar phase is drawn. The distance between the centers of the stacks is on the order of the wavelength of light. When light with a certain angle of

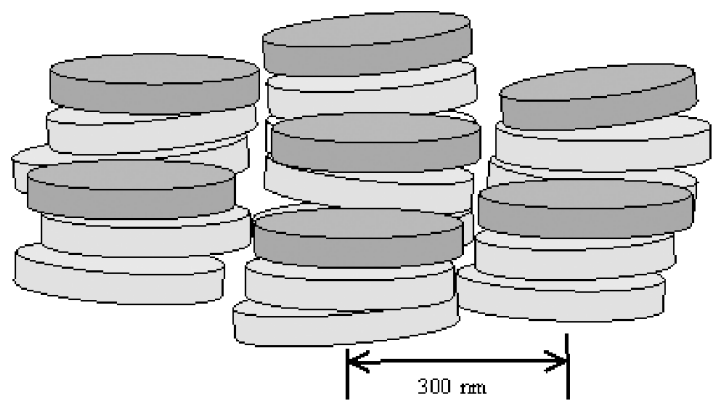

(a)

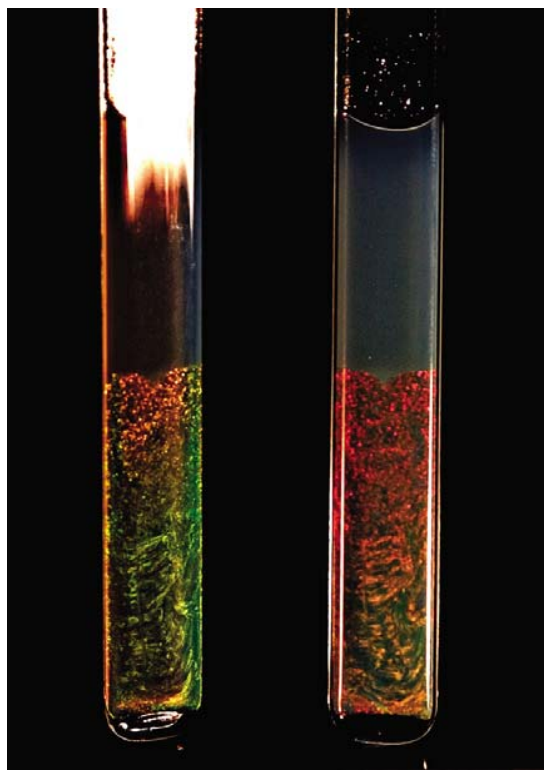

(b)

Fig. 7. (a) The columnar phase consisting of stacks of platelets. The distance between the stacks is on the order of the wavelength of light. (b) Bright Bragg reflections of the same sample at different angles of incidence. Due to the low polydispersity, the quality of the columnar phase is very good. Particle diameter is $270 \mathrm{~nm} \pm 18 \%$. 


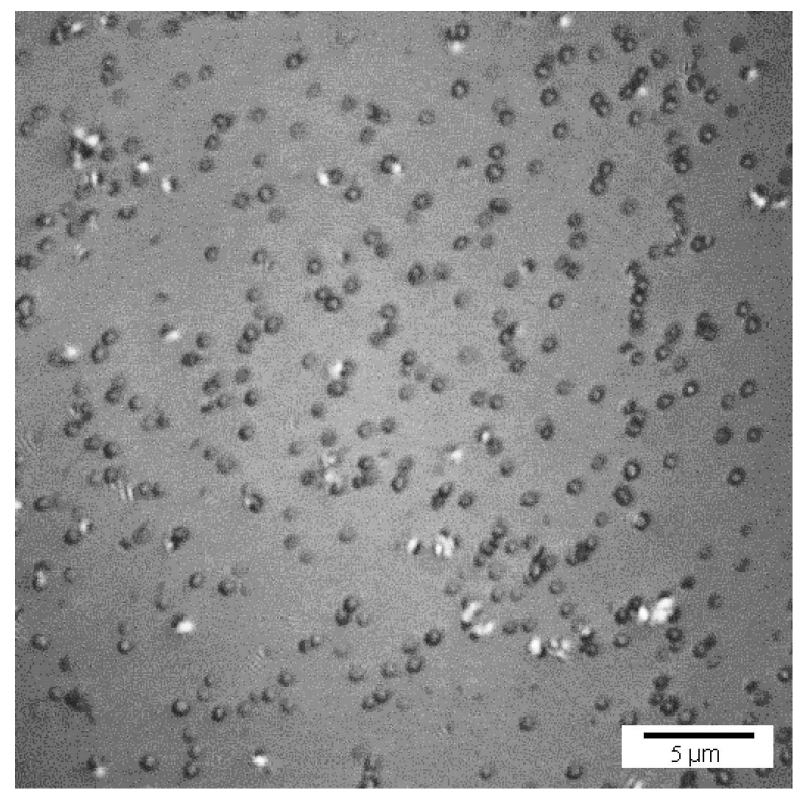

Fig. 8. Confocal microscopy image of gibbsite plates of $570 \mathrm{~nm}$ (in reflection mode). Individual plates are clearly visible.

incidence $(\theta)$ enters the columnar phase it is reflected when it obeys Bragg's law

$2 d \sin \theta=n \lambda$,

in which $d$ is the distance between the centers of the columns, $n$ is an integer, and $\lambda$ is the wavelength. Because it is expected that the formation of a columnar phase is enhanced by a low polydispersity, we concentrated a sample of low polydispersity plates to the volume fraction of particles where columnar phases are expected: $200 \mathrm{~g} / \mathrm{L}$ at an ionic strength of $10^{-3} \mathrm{M} \mathrm{NaCl}$. The columnar phase is formed within a few days. Bright Bragg reflections from the columnar phase can be seen in that sample (Fig. 7b), many crystallites are visible. The color of the reflection is depending on the angle of incidence of the light. From a white light source red and green Bragg reflections are obtained (among others).

The particles from the last step of the seeded growth are large enough $(570 \mathrm{~nm})$ to be visible in a light microscope. Fig. 8 shows a picture taken with a confocal microscopy in reflection mode. All particles are visible as individual reflections, their shape cannot be determined; the particles are too small for that.

\section{Conclusion}

Starting from gibbsite seeds obtained by heating of an aluminum alkoxide solution, gibbsite colloids of different sizes can be obtained. There was no sign of a limit of the seeded growth in the region studied $(150-570 \mathrm{~nm})$. Smaller particles with a lower size limit of $90 \mathrm{~nm}$ can be obtained by growth at room temperature. The polydispersity of the particles is reduced by centrifugation and by continued growth. The planes of gibbsite particles grow at different rates so the aspect ratio of the particles varies. The growth rates can be influenced by growth circumstances. With low polydispersity particles a columnar phase can be more easily formed, as expected. The large particles can be made visible with a confocal microscope in reflection mode.

\section{Acknowledgments}

Daniëlle van't Zand is thanked for performing AFM measurements. Dirk Aarts helped with the confocal microscope. Marjan Versluys-Helder (Inorganic Chemistry, Utrecht University), Andrei Petukhov, Andries Meijerink, and Wouter van Beek (BM-1, ESRF) are thanked for performing X-ray diffraction and help with the interpretation. Access to the beamline BM-1 was made possible in the framework of the DUBBLE, Swiss-Norwegian collaboration. Professor Henk Lekkerkerker and Martijn Oversteegen read the manuscript carefully. This work was financially supported by the "Nederlandse organisatie voor Wetenschappelijk Onderzoek" (NWO).

\section{References}

[1] J.C.P. Gabriel, P. Davidson, in: M. Antonietti (Ed.), Colloid Chemistry 1, vol. 226, Springer, Berlin, 2003, pp. 119-172.

[2] A.R. Hind, S.K. Bhargava, S.C. Grocott, Colloids Surf. A Physicochem. Eng. Aspects 146 (1999) 359-374.

[3] M.Y. Lee, G.M. Parkinson, P.G. Smith, F.J. Lincoln, M.M. Reyhani, in: G.D. Botsaris, K. Toyokura (Eds.), Separation and Purification by Crystallization, vol. 667, Am. Chem. Soc., Washington, 1997, pp. 123-133.

[4] C. Sweegers, H.C. de Coninck, H. Meekes, W.J.P. van Enckevoort, I.D.K. Hiralal, A. Rijkeboer, J. Cryst. Growth 233 (2001) 567-582.

[5] Altech, The world of alumina at http://www.altech.pechiney.com.

[6] Martinswerk, http://www.martinswerk.de.

[7] F.M. van der Kooij, H.N.W. Lekkerkerker, E.S. Boek, UK patent application GB2378716A, International Publication Number WO03/014252A1, 2003.

[8] A.L. Gimsing, O.K. Borggaard, P. Sestoft, Environ. Sci. Technol. 38 (2004) 1718-1722.

[9] J. Rosenqvist, P. Persson, S. Sjoberg, Langmuir 18 (2002) 4598-4604.

[10] G. Lefevre, M. Duc, P. Lepeut, R. Caplain, M. Fedoroff, Langmuir 18 (2002) 7530-7537.

[11] T. Hiemstra, H. Yong, W.H. van Riemsdijk, Langmuir 15 (1999) 5942 5955.

[12] Y.-P. Lee, Y.-H. Liu, C.-S. Yeh, Phys. Chem. Chem. Phys. 1 (1999) 4681-4686.

[13] D.H. Rasmussen, C. Brancewicz, B. Das, M. Graeffe, J. Rosenholm, A. Toscano, J. Dispers. Sci. Technol. 22 (2001) 491-498.

[14] M.C. Gastuche, A. Herbillon, Bull. Soc. Chim. (1962) 1404-1412.

[15] N. Phambu, B. Humbert, A. Burneau, Langmuir 16 (2000) 6200-6207.

[16] A.P. Philipse, A.-M. Nechifor, C. Patmamanoharan, Langmuir 10 (1994) 4451-4458.

[17] A.M. Wierenga, T.A.J. Lenstra, A.P. Philipse, Colloids Surf. A Physicochem. Eng. Aspects 134 (1998) 359-371.

[18] F.M. van der Kooij, A.P. Philipse, J.K.G. Dhont, Langmuir 16 (2000) $5317-5323$. 
[19] F.M. van der Kooij, E.S. Boek, A.P. Philipse, J. Colloid Interface Sci. 235 (2001) 344-349.

[20] F.M. van der Kooij, H.N.W. Lekkerkerker, Phys. Rev. Lett. 84 (2000) 781-784.

[21] F.M. van der Kooij, H.N.W. Lekkerkerker, J. Phys. Chem. B 102 (1998) 7829-7832.

[22] D. van der Beek, H.N.W. Lekkerkerker, Europhys. Lett. 61 (2003) 702-707.

[23] M.P.B. van Bruggen, Langmuir 14 (1998) 2245-2255.
[24] A.V. Blaaderen, A. Vrij, Langmuir 8 (1992) 2921-2931.

[25] F.M. van der Kooij, D. van der Beek, unpublished results.

[26] H. Giesche, J. Eur. Ceram. Soc. 14 (1994) 205-214.

[27] G.H. Bogush, M.A. Tracy, C.F. Zukoski, J. Non-Cryst. Solids 104 (1988) 95-106.

[28] C. Pathmamanoharan, N.L. Zuiverloon, A.P. Philipse, Prog. Colloid Polym. Sci. 115 (2000) 141-145.

[29] J.E.G.J. Wijnhoven, D. van der Beek, D. van't Zand, H.N.W. Lekkerkerker, submitted for publication. 